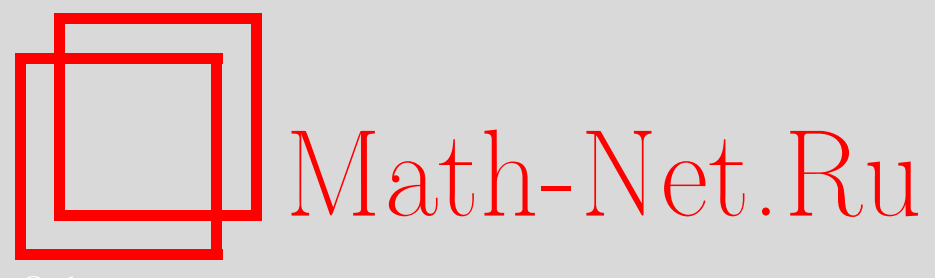

С. А. Степанов, Векторные инварианты симметрических групп в случае поля простой характеристики, Дискрет. матем., 2000, том 12, выпуск 4, 25-38

DOI: https://doi.org/10.4213/dm356

Использование Общероссийского математического портала Math-Net.Ru подразумевает, что вы прочитали и согласны с пользовательским соглашением http: //www.mathnet.ru/rus/agreement

Параметры загрузки:

IP : 54.198 .67 .100

26 апреля 2023 г., 11:25:22 


\title{
Векторные инварианты симметрических групп в случае поля простой характеристики
}

\author{
(c) 2000 г. С. А. Степанов
}

Пусть $R$ - коммутативное кольцо с единичным элементом 1 и $S_{n}-$ симметрическая группа степени $n \geqslant 1$. Обозначим $A_{m n}^{S_{n}}$ подалгебру инвариантов алгебры полиномов

$$
A_{m n}=R\left[x_{11}, \ldots, x_{1 n} ; \ldots ; x_{m 1}, \ldots, x_{m n}\right]
$$

относительно $S_{n}$. Согласно классическому результату Г. Вейля, если каждое не равное нулю целое число обратимо в $R$, то алгебра $A_{m n}^{S_{n}}$ порождается поляризованными элементарными симметрическими полиномами степеней, не превосходящих $n$, вне зависимости от величины $m$. Как недавно было доказано Ричменом, этот результат остается справедливым, если число $\left|S_{n}\right|=n$ ! обратимо в $R$. С другой стороны, для случая, когда $R$ - поле простой характеристики $p \leqslant n$, Ричменом доказано, что каждая система образующих $R$-алгебры $A_{m n}^{S_{n}}$, содержит элемент, степень которого не меньше, чем $\max \{n,(m+p-n) /(p-1)\}$. Из этого результата следует, что предложенная Вейлем оценка сверху степеней порождающих элементов перестает быть верной в случае, когда характеристика $p$ поля $R$ делит $\left|S_{n}\right|$. В общем случае доказано, что для произвольного коммутативного кольца $R$ алгебра $A_{m n}^{S_{n}}$ порождается инвариантами степени, не превосходящей $\max \{n, m n(n-1) / 2\}$. Цель этой статьи состоит в том, чтобы дать простое арифметическое доказательство первого результата Ричмена и усилить его второй результат, также используя новые арифметические аргументы. Независимо аналогичное усиление результата Ричмена о нижняя оценке было предложено Кемпером с использованием другого подхода. Недавний результат Флейшмана показывает, что полученная в статье нижняя оценка является точной, если $m>1$ и $n=p^{\alpha}$, где $p$ - простое число.

\section{1. Введение}

Пусть $m, n$ - положительные целые числа, $R$ - коммутативное кольцо с единичным элементом 1 и

$$
A_{m n}=R\left[x_{11}, \ldots, x_{m 1} ; \ldots ; x_{1 n}, \ldots, x_{m n}\right]
$$

- алгебра полиномов от $m n$ переменных $x_{i j}$ над кольцом $R$. Симметрическая группа $S_{n}$ действует на алгебре $A_{m n}$ как группа $R$-автоморфизмов по правилу

$$
\sigma\left(x_{i j}\right)=x_{i, \sigma(j)}, \quad \sigma \in S_{n} .
$$


Обозначим $A_{m n}^{S_{n}}$ подалгебру инвариантов алгебры $A_{m n}$ относительно группы $S_{n}$ и определим поляризованные элементарные полиномы $u_{r_{1}, \ldots, r_{m}} \in A_{m n}^{S_{n}}$ от $n$ векторных переменных

$$
\left(x_{11}, \ldots, x_{m 1}\right), \ldots,\left(x_{1 n}, \ldots, x_{m n}\right)
$$

с помощью формального тождества

$$
\prod_{j=1}^{n}\left(1+x_{1 j} z_{1}+\ldots+x_{m j} z_{m}\right)=1+\sum_{1 \leqslant r_{1}+\ldots+r_{m} \leqslant n} u_{r_{1}, \ldots, r_{m}} z_{1}^{r_{1}} \ldots z_{m}^{r_{m}} .
$$

Элементы алгебры $A_{m n}^{S_{n}}$ обычно называются векторными инвариантами относительно $S_{n}$. Если кольцо $R$ нетерово, то по теореме Гильберта-Нетер (см. [6, 9]) алгебра $A_{m n}^{S_{n}}$ - конечно порожденная коммутативная $R$-алгебра и алгебра $A_{m n}$ конечно порождаема как модуль над $A_{m n}^{S_{n}}$. Более того, если каждое ненулевое целое число обратимо в $R$, то инварианты $u_{r_{1}, \ldots, r_{m}}$ составляют полную систему образующих алгебры $A_{m n}^{S_{n}}$ над $R$ (см. [1], с.9; [17], с.37). Другими словами, каждый элемент $u$ алгебры $A_{m n}^{S_{n}}$ может быть записан в виде полинома от

$$
u_{r_{1}, \ldots, r_{m}}, \quad 1 \leqslant r_{1}+\ldots+r_{m} \leqslant n
$$

с коэффициентами из $R$. Приведенная выше система порождающих инвариантов содержит $\left(\begin{array}{c}m+n \\ m\end{array}\right)-1$ элементов, связанных между собой различными алгебраическими соотношениями (см. [4], с. 68, и [14]). Этот результат недавно был обобщен Ричменом следующим образом (см. [11]).

Теорема 1. Предположим, что $\left|S_{n}\right|=n$ ! обратимо в $R$. Тогда $A_{m n}^{S_{n}}$ порождается как $R$-алгебра поляризованными элементарными полиномами

$$
u_{r_{1}, \ldots, r_{m}}, \quad 1 \leqslant r_{1}+\ldots+r_{m} \leqslant n
$$

степени, не большей $n$.

В частности, если $R$ - поле простой характеристики $p>n$, то $n$ ! обратимо в $R$, так что справедлив следующий результат.

Следствие 1. Пусть $R-$ поле. Если $\operatorname{char} R=0$ или $\operatorname{char} R=p>n$, mo $A_{m n}^{S_{n}}$ порождается как $R$-алгебра поляризованными элементарными симметрическими полиномами

$$
u_{r_{1}, \ldots, r_{m}}, \quad 1 \leqslant r_{1}+\ldots+r_{m} \leqslant n .
$$

В этой статье приводится простое арифметическое доказательство теоремы 1 , опирающееся на поляризацию классической формулы Варинга [16] и тесно связанное с первоначальным доказательством Вейля [17] в случае, когда $R$ содержит поле рациональных чисел $\mathbf{Q}$.

Теорему 1 можно легко обобщить следующим образом. Пусть

$$
A=R\left[x_{1}, \ldots, x_{m}\right]
$$

- конечно порождаемая коммутативная $R$-алгебра, $G$ - конечная группа $R$-алгебры автоморфизмов $A$ и $A^{G}$ - подалгебра инвариантов группы $G$. Для коммутирующих переменных $z_{1}, \ldots, z_{m}$ положим

$$
F\left(z_{1}, \ldots, z_{m}\right)=\prod_{\sigma \in G}\left(1+\sigma\left(x_{1}\right) z_{1}+\sigma\left(x_{2}\right) z_{2}+\ldots+\sigma\left(x_{m}\right) z_{m}\right) .
$$


Если каждое целое число, не равное нулю, обратимо в $R$, то из теоремы Нетер следует, что $A^{G}$ порождается как $R$-алгебра коэффициентами полинома $F\left(z_{1}, \ldots, z_{m}\right)$. Теорема 1 и стандартные рассуждения с использованием оператора Рейнольдса и отображения Нетер (см. [8]; [12], с. 63; [17], с. 275) приводят к следующему утверждению.

Теорема 2. Eсли $|G|$ ! обратимо в $R$, то $A^{G}$ порождается как $R$-алгебра коэффициентами полинома $F\left(z_{1}, \ldots, z_{m}\right)$. Другими словами, $A^{G}$ порождается над $R$ инвариантнъми полиномами от $x_{1}, \ldots, x_{m}$ степени, не болъшей $|G|$.

Теоремы 1 и 3 позволяют построить эффективный алгоритм вычисления полной системы порождающих полиномиальных инвариантов при условии, что $|G|$ ! обратимо в $R$. Существует другое конструктивное доказательство теоремы 3 , опирающееся на рассуждения, также восходящие к Нетер (см. [12], с. 29). Оценка сверху для степеней множества порождающих полиномов для алгебры инвариантов, приведенная в теореме 3, известна как граница Нетер (см. также [12], с. 28, и [13]).

Если $\left|S_{n}\right|=n$ ! обратимо в $R$, то верхняя оценка степеней образующих $R$-алгебры $A_{m n}^{S_{n}}$, приведенная в теореме 1 , является неулучшаемой. Для случая, когда $R-$ произвольное коммутативное кольцо, в [3] доказано, что алгебра Вейля $A_{m n}^{S_{n}}$ порождается над $R$ полиномами, степени которых не превышают $\max \{n, m n(n-1) / 2\}$. Аналогичный результат был также получен Ричменом (см. [11], предл. 7). С другой стороны, в настоящей статье дана следующая оценка снизу.

Теорема 3. Пусть $\alpha-$ положительное челое число, $S_{n}$ - симметрическал группа степени $n \geqslant 2$ и $R$ - поле простой характеристики $p$. Если $p^{\alpha}$ делит $n$, то любая система образующих $R$-алгебры $A_{m n}^{S_{n}}$ содержит элемент, степень которого не менъше, чем $\max \left\{n, m\left(p^{\alpha}-1\right)\right\}$.

Этот результат улучшает приведенную выше нижнюю оценку, предложенную Ричменом, в случае, когда $p$ - простой делитель $n$, и показывает, что верхняя оценка, предложенная Нетер, неверна, если $n$ необратимо в $R$. Как недавно было доказано Флейшманом [5], нижняя оценка теоремы 3 неулучшаема, если $n=p^{\alpha}$ и $m>1$.

Теорема 3 может быть легко обобщена следующим образом. Пусть $r$ - положительное целое число, не превосходящее $n$. В этом случае группа $S_{r}$ является подгруппой $S_{n}$ и поэтому $A_{m n}^{S_{n}} \subseteq A_{m n}^{S_{r}}$. Это замечание и теорема 3 , примененная к $S_{r}$, приводят к следующему результату.

Следствие 2. Пусть $\lambda u r \leqslant n-$ положительные целые иисла, $S_{n}$ - симметрическая группа степени $n \geqslant 2$ и $R$ - поле простой характеристики $p$. Если $p^{\lambda}$ делит $r$, то каждая система образующих $R$-алгебры $A_{m n}^{S_{n}}$ содержит элемент, степень которого не меньше, чем $\max \left\{n, m\left(p^{\lambda}-1\right)\right\}$.

Следствие 3. Пусть $S_{n}$ - симметрическая группа степени $n \geqslant 2 u R-$ коммутативное колъцо с единичным элементом 1. Если $p$ - простой делитель $n$ !, необратимый в $R$, то каждая система образующих $R$-алгебры $A_{m n}^{S_{n}}$ содержит элемент, степенъ которого не менъше, чем $\max \{n, m(p-1)\}$.

Если $R$ совпадает с кольцом $\mathbf{Z}$ целых чисел, то для получения универсальной нижней оценки, зависящей от $m$ и $n$, можно использовать известные результаты о распределении простых чисел в коротких интервалах. 
Следствие 4. Пусть $R=\mathrm{Z}$ - кольцо целъх чисел $и S_{n}$ - симметрическая группа степени $n \geqslant 2$. Тогда каждая система образующих $R$-алгебры $A_{m n}^{S_{n}}$ содержит элемент, степенъ которого не менъие, чем $\max \left\{n, c_{n} m(n-1)\right\}$, где $c_{n}=1 / 2$ для любых $n \geqslant 2$; кроме того, $c_{n}=5 / 6$ для $n \geqslant 25 u c_{n} \rightarrow 1$ при $n \rightarrow \infty$. В частности, если $n$ - простое число, то каждая система образующих $R$-алгебры $A_{m n}^{S_{n}}$ содержит элемент, степенъ которого не менъше, чем $\max \{n, m(n-1)\}$.

Аналогичный результат справедлив в случае, когда $R-$ кольцо $\mathbf{Z}_{K}$ целых чисел числового поля $K$.

\section{2. Порождающие инварианты симметрической группы}

Пусть $S_{n}-$ симметрическая группа степени $n \geqslant 1$, действующая на $R$-алгебре

$$
A_{m n}=R\left[x_{11}, \ldots, x_{m 1} ; \ldots ; x_{1 n}, \ldots, x_{m n}\right]
$$

как группа $R$-автоморфизмов, $A_{m n}^{S_{n}}$ - подалгебра инвариантов алгебры $A_{m n}$ относительно $S_{n}$ и

$$
u_{r_{1}, \ldots, r_{m}}, \quad 1 \leqslant r_{1}+\ldots+r_{m} \leqslant n,
$$

- поляризованные элементарные симметрические полиномы в $A_{m n}^{S_{n}}$.

Пусть $v_{s_{1}, \ldots, s_{m}}$ - инвариантный полином в $A_{m n}^{S_{n}}$, имеющий вид

$$
v_{s_{1}, \ldots, s_{m}}=\sum_{j=1}^{n} x_{1 j}^{s_{1}} \ldots x_{m j}^{s_{m}}
$$

Если $m=1$, то согласно известной формуле Варинга (см. [16], стр. 13, и [2], стр. 99)

$$
v_{s}=\sum_{j=1}^{n} x_{j}^{s}=\sum_{k_{1}+2 k_{2}+\ldots+n k_{n}=s} c\left(k_{1}, \ldots, k_{n}\right) u_{1}^{k_{1}} \ldots u_{n}^{k_{n}}
$$

где $c\left(k_{1}, \ldots, k_{n}\right)$ - целые числа вида

$$
c\left(k_{1}, \ldots, k_{n}\right)=(-1)^{k_{2}+2 k_{3}+\ldots+(n-1) k_{n}} \frac{\left(k_{1}+\ldots+k_{n}-1\right) ! s}{k_{1} ! \ldots k_{n} !} .
$$

Следующий результат можно рассматривать как обобщение формулы Варинга на случай, когда $m>1$ (см. также $[14,15])$.

Предложение 1. Пусть $s_{1}, \ldots, s_{m}$ - неотрицателъные целье числа,

$$
v_{s_{1}, \ldots, s_{m}}=\sum_{j=1}^{n} x_{1 j}^{s_{1}} \ldots x_{m j}^{s_{m}}
$$

- полином в $A_{m n}^{S_{n}}$ степени $s=s_{1}+\ldots+s_{m} u$

$$
u_{r_{1}, \ldots, r_{m}}, \quad 1 \leqslant r_{1}+\ldots+r_{m} \leqslant n
$$


- поляризованные элементарные симметрические полиномы от векторов

$$
\left(x_{1 j}, \ldots, x_{m j}\right), \quad 1 \leqslant j \leqslant n .
$$

Для неотрицательных целых чисел $k_{1}, \ldots, k_{n} u k_{1 \nu}, \ldots, k_{m \nu}$ таких, что

$$
\begin{aligned}
k_{1}+2 k_{2}+\ldots+n k_{n} & =s, \\
k_{1 \nu}+\ldots+k_{m \nu} & =\nu k_{\nu}, \quad 1 \leqslant \nu \leqslant n,
\end{aligned}
$$

nycms

$$
w_{k_{1 \nu}, \ldots, k_{m \nu}}=\sum_{A_{m, n}} \frac{k_{\nu} !}{l_{\nu 1} ! \ldots l_{\nu k_{\nu}} !} \prod_{\mu=1}^{k_{\nu}} u_{r_{1 \mu}, \ldots, r_{m \mu}}^{l_{\nu \mu}}
$$

где сумма берется по множеству $A_{m, n}$ всех неотрицателъных целых чисел $r_{1 \mu}, \ldots, r_{m \mu}$ u $l_{\nu 1}, \ldots, l_{\nu k_{\nu}} \operatorname{maxux,~чmo~}$

$$
\begin{aligned}
r_{i 1} l_{\nu 1}+\ldots+r_{i k_{\nu}} l_{\nu k_{\nu}} & =k_{i \nu} \\
l_{\nu 1}+\ldots+l_{\nu k_{\nu}} & =k_{\nu} \\
r_{1 \mu}+\ldots+r_{m \mu} & =\nu
\end{aligned}
$$

npu $1 \leqslant i \leqslant m, 1 \leqslant \mu \leqslant k_{\nu}, 1 \leqslant \nu \leqslant n$.

Тогда

$$
v_{s_{1}, \ldots, s_{m}}=\frac{s_{1} ! \ldots s_{m} !}{s !} \sum_{k_{1}+2 k_{2}+\ldots+n k_{n}=s} c\left(k_{1}, \ldots, k_{n}\right) \sum_{B_{m, n}} \prod_{\nu=1}^{n} w_{k_{1 \nu}, \ldots, k_{m \nu}}
$$

где $s=s_{1}+\ldots+s_{m}$ и внутренняя сумма берется по множеству $B_{m, n}$ всех целых неотрицателъных чисел $k_{i 1}, \ldots, k_{\text {in }}$ таких, что

$$
\begin{aligned}
k_{i 1}+\ldots+k_{i n} & =s_{i}, \\
k_{1 \nu}+\ldots+k_{m \nu} & =\nu k_{\nu}
\end{aligned}
$$

npu $1 \leqslant i \leqslant m, 1 \leqslant \nu \leqslant n$.

Доказательство. Положим в (1)

$$
x_{j}=x_{1 j} z_{1}+\ldots+x_{m j} z_{m}, \quad 1 \leqslant j \leqslant n .
$$

Поскольку

$$
\left(x_{1 j} z_{1}+\ldots+x_{m j} z_{m}\right)^{s}=\sum_{s_{1}+\ldots+s_{m}=s} \frac{s !}{s_{1} ! \ldots s_{m} !} x_{1 j}^{s_{1}} \ldots x_{m j}^{s_{m}} z_{1}^{s_{1}} \ldots z_{m}^{s_{m}}
$$

справедливо равенство

$$
\sum_{j=1}^{n}\left(x_{1 j} z_{1}+\ldots+x_{m j} z_{m}\right)^{s}=\sum_{s_{1}+\ldots+s_{m}=s} \frac{s !}{s_{1} ! \ldots s_{m} !}\left(\sum_{j=1}^{n} x_{1 j}^{s_{1}} \ldots x_{m j}^{s_{m}}\right) z_{1}^{s_{1}} \ldots z_{m}^{s_{m}} .
$$

С другой стороны,

$$
\sum_{1 \leqslant j_{1}<\ldots<j_{\nu} \leqslant n} \prod_{k=1}^{\nu}\left(x_{1 j_{k}} z_{1}+\ldots+x_{m j_{k}} z_{m}\right)=\sum_{r_{1}+\ldots+r_{m}=\nu} u_{r_{1}, \ldots, r_{m}} z_{1}^{r_{1}} \ldots z_{m}^{r_{m}}
$$


и следовательно, в силу (1)

$$
\begin{aligned}
\sum_{j=1}^{n}\left(x_{1 j} z_{1}+\ldots+x_{m j} z_{m}\right)^{s} & \\
& =\sum_{k_{1}+2 k_{2}+\ldots+n k_{n}=s} c\left(k_{1}, \ldots, k_{n}\right) \prod_{\nu=1}^{n}\left(\sum_{r_{1}+\ldots+r_{m}=\nu} u_{r_{1}, \ldots, r_{m}} z_{1}^{r_{1}} \ldots z_{m}^{r_{m}}\right)^{k_{\nu}} .
\end{aligned}
$$

В результате получаем, что

$$
\begin{aligned}
\sum_{s_{1}+\ldots+s_{m}=s} & \frac{s !}{s_{1} ! \ldots s_{m} !}\left(\sum_{j=1}^{n} x_{1 j}^{s_{1}} \ldots x_{m j}^{s_{m}}\right) z_{1}^{s_{1}} \ldots z_{m}^{s_{m}} \\
& =\sum_{k_{1}+2 k_{2}+\ldots+n k_{n}=s} c\left(k_{1}, \ldots, k_{n}\right) \prod_{\nu=1}^{n}\left(\sum_{r_{1}+\ldots+r_{m}=\nu} u_{r_{1}, \ldots, r_{m}} z_{1}^{r_{1}} \ldots z_{m}^{r_{m}}\right)^{k_{\nu}} .
\end{aligned}
$$

Теперь, поскольку

$$
\left(\sum_{r_{1}+\ldots+r_{m}=\nu} u_{r_{1}, \ldots, r_{m}} z_{1}^{r_{1}} \ldots z_{m}^{r_{m}}\right)^{k_{v}}=\sum_{k_{1 \nu}+\ldots+k_{m \nu}=\nu k_{\nu}} w_{k_{1 \nu}, \ldots, k_{m \nu}} z_{1}^{k_{1 \nu}} \ldots z_{m}^{k_{m \nu}}
$$

где

$$
w_{k_{1 \nu}, \ldots, k_{m \nu}}=\sum_{C_{m, \nu}} \frac{k_{\nu} !}{l_{\nu 1} ! \ldots l_{\nu k_{\nu}} !} \prod_{\mu=1}^{k_{\nu}} u_{r_{1 \mu}, \ldots, r_{m \mu}}^{l_{\nu \mu}}
$$

и суммирование проводится по множеству $C_{m, \nu}$ всех неотрицательных целых чисел $r_{1 \mu}, \ldots, r_{m \mu}$ и $l_{\nu 1}, \ldots, l_{\nu k_{\nu}}$ таких, что

$$
\begin{array}{rlrl}
r_{i 1} l_{\nu 1}+\ldots+r_{i s_{\nu}} l_{\nu k_{\nu}} & =k_{i \nu}, & & 1 \leqslant i \leqslant m \\
r_{1 \mu}+\ldots+r_{m \mu} & =\nu, & & 1 \leqslant \mu \leqslant k_{\nu} \\
l_{\nu 1}+\ldots+l_{\nu k_{\nu}} & =k_{\nu}, &
\end{array}
$$

находим, что

$$
\begin{aligned}
& \sum_{s_{1}+\ldots+s_{m}=s} \frac{s !}{s_{1} ! \ldots s_{m} !}\left(\sum_{j=1}^{n} x_{1 j}^{s_{1}} \ldots x_{m j}^{s_{m}}\right) z_{1}^{s_{1}} \ldots z_{m}^{s_{m}} \\
& \quad=\sum_{s_{1}+\ldots+s_{m}=s}\left(\sum_{k_{1}+2 k_{2}+\ldots+n k_{n}=s} c\left(k_{1}, \ldots, k_{n}\right) \sum_{B_{m, n}} \prod_{\nu=1}^{n} w_{k_{1 \nu}, \ldots, k_{m \nu}}\right) z_{1}^{s_{1}} \ldots z_{m}^{s_{m}}
\end{aligned}
$$

где суммирование в последней сумме проводится по множеству $B_{m, n}$ всех неотрицательных целых чисел $k_{i 1}, \ldots, k_{i \nu}, i=1, \ldots, m$, таких, что

$$
\begin{aligned}
k_{i 1}+\ldots+k_{i \nu} & =s_{i}, & & 1 \leqslant i \leqslant m \\
k_{1 \nu}+\ldots+k_{m \nu} & =\nu k_{\nu}, & & 1 \leqslant \nu \leqslant n .
\end{aligned}
$$


Таким образом, приходим к соотношению

$$
\sum_{j=1}^{n} x_{1 j}^{s_{1}} \ldots x_{m j}^{s_{m}}=\frac{s_{1} ! \ldots s_{m} !}{s !} \sum_{k_{1}+2 k_{2}+\ldots+n k_{n}=s} c\left(k_{1}, \ldots, k_{n}\right) \sum_{B_{m, n}} \prod_{\nu=1}^{n} w_{k_{1 \nu}, \ldots, k_{m \nu}},
$$

что и доказывает предложение.

$$
\begin{aligned}
& \text { Если } s=s_{1}+\ldots+s_{m} \leqslant n+1 \text {, то } \\
& \qquad v_{s_{1}, \ldots, s_{m}}=\frac{s_{1} ! \ldots s_{m} !}{s !} \sum_{k_{1}+2 k_{2}+\ldots+n k_{n}=s} c\left(k_{1}, \ldots, k_{n}\right) \sum_{B_{m, n}} \prod_{\nu=1}^{n} w_{k_{1 \nu}, \ldots, k_{m \nu}}
\end{aligned}
$$

есть полином от $u_{r_{1}, \ldots, r_{m}}, 1 \leqslant r_{1}+\ldots+r_{m} \leqslant n$, с рациональными коэффициентами, знаменатели которых не делятся ни на одно простое число $p \geqslant n+1$. С помощью этого замечания получаем следующее утверждение.

Следствие 5. Если $n$ ! обратимо в $R$ u $s=s_{1}+\ldots+s_{m} \leqslant n+1, m o$

$$
v_{s_{1}, \ldots, s_{m}}=\sum_{j=1}^{n} x_{1 j}^{s_{1}} \ldots x_{m j}^{s_{m}}
$$

есть полином над $R$ от $u_{r_{1}, \ldots, r_{m}}, 1 \leqslant r_{1}+\ldots+r_{m} \leqslant n$, степенъ которого не болъше, чем $n$.

Покажем теперь, что если $n$ ! обратимо в $R$, то любой векторный инвариант в $A_{m n}^{S_{n}}$ можно представить как полином над $R$ от инвариантов $v_{s_{1}, \ldots, s_{m}}$.

Предложение 2. Пусть $f-$ одночлен в $A_{m n} u$

$$
v=\sum_{u \in\left\{\sigma(f) \mid \sigma \in S_{n}\right\}} u \text {. }
$$

Eсли $n$ ! обратимо в $R$, то $v$ - полином над $R$ от инвариантов

$$
v_{s_{1}, \ldots, s_{m}}=\sum_{j=1}^{n} x_{1 j}^{s_{1}} \ldots x_{m j}^{s_{m}}
$$

где $s_{1}, \ldots, s_{m}$ - неотрицательные целье числа такие, что

$$
0 \leqslant s_{1}+\ldots+s_{m} \leqslant \operatorname{deg} f
$$

Доказательство. Запишем $f$ в виде $f=f_{1} \ldots f_{n}$, где каждый множитель $f_{j}-$ одночлен в $R\left[x_{1 j}, \ldots, x_{m j}\right]$. Положим

$$
d(f)=\max _{1 \leqslant j \leqslant n}\left(\operatorname{deg} f_{j}\right)
$$

и докажем предложение 2 индукцией по $\delta(f)=\operatorname{deg} f-d(f)$. Предположим вначале, что $\delta(f)=0$. Тогда

$$
f=f_{j}=x_{1 j}^{s_{1}} \ldots x_{m j}^{s_{m}}
$$


где $j \in\{1,2, \ldots, n\}, s_{1}, \ldots, s_{m}-$ натуральные числа, $s_{1}+\ldots+s_{m}=\operatorname{deg} f$, и

$$
v=\sum_{u \in\left\{\sigma(f) \mid \sigma \in S_{n}\right\}} u=\sum_{j=1}^{n} x_{1 j}^{s_{1}} \ldots x_{m j}^{s_{m}} .
$$

Предположим теперь, что $\delta(f)>0$, и пусть $j \in\{1,2, \ldots, n\}$ таково, что

$$
d(f)=\operatorname{deg} f_{j}<\operatorname{deg} f .
$$

Определим $v_{j}$ и $v_{j}^{\prime}$, полагая

$$
v_{j}=\sum_{u \in\left\{\sigma\left(f_{j}\right) \mid \sigma \in S_{n}\right\}} u, \quad v_{j}^{\prime}=\sum_{u^{\prime} \in\left\{\sigma\left(f / f_{j}\right) \mid \sigma \in S_{n}\right\}} u^{\prime} .
$$

По предположению индукции $v_{j}$ и $v_{j}^{\prime}-$ полиномы от

$$
v_{s_{1}, \ldots, s_{m}}, \quad 0 \leqslant s_{1}+\ldots+s_{m} \leqslant \operatorname{deg} f .
$$

Для каждого $\tau \in S_{n}$ пусть $U_{\tau}-$ множество всех пар $\left(u, u^{\prime}\right)$ таких, что

$$
u \in\left\{\sigma\left(f_{j}\right) \mid \sigma \in S_{n}\right\}, \quad u^{\prime} \in\left\{\sigma\left(f / f_{j}\right) \mid \sigma \in S_{n}\right\}, \quad u u^{\prime}=\tau(f),
$$

и заметим, что отображение

$$
U_{i d} \rightarrow U_{\tau}, \quad\left(u, u^{\prime}\right) \rightarrow\left(\tau(u), \tau\left(u^{\prime}\right)\right)
$$

является биекцией. Таким образом, $\left|U_{\tau}\right|=\left|U_{i d}\right|$ для всех $\tau \in S_{n}$. Заметим также, что $d\left(u u^{\prime}\right) \geqslant d(f)$ для всех $u \in\left\{\sigma\left(f_{j}\right) \mid \sigma \in S_{n}\right\}$ и $u^{\prime} \in\left\{\sigma\left(f / f_{j}\right) \mid \sigma \in S_{n}\right\}$, где равенство достигается тогда и только тогда, когда $u u^{\prime} \in\left\{\sigma(f) \mid \sigma \in S_{n}\right\}$. Поэтому

$$
v_{j} v_{j}^{\prime}=\left|U_{i d}\right| \sum_{u \in\left\{\sigma(f) \mid \sigma \in S_{n}\right\}} u+\sum_{f^{\prime}: \operatorname{deg} f^{\prime}=\operatorname{deg} f, d\left(f^{\prime}\right)>d(f)} \sum_{u \in\left\{\sigma\left(f^{\prime}\right) \mid \sigma \in S_{n}\right\}} u .
$$

По предположению индукции инвариант

$$
v_{j} v_{j}^{\prime}-\left|U_{i d}\right| \sum_{u \in\left\{\sigma(f) \mid \sigma \in S_{n}\right\}} u=\sum_{f^{\prime}: \operatorname{deg} f^{\prime}=\operatorname{deg} f, d\left(f^{\prime}\right)>d(f)} \sum_{u \in\left\{\sigma\left(f^{\prime}\right) \mid \sigma \in S_{n}\right\}} u
$$

есть полином над $R$ от $v_{s_{1}, \ldots, s_{m}}, 0 \leqslant s_{1}+\ldots+s_{m} \leqslant \operatorname{deg} f$. Мощность $U_{i d}$ не превосходит мощности $\left\{\sigma\left(f_{j}\right) \mid \sigma \in S_{n}\right\}$, которая в свою очередь не превосходит мощности $\left\{x_{1 j}^{s_{1}} \ldots x_{m j}^{s_{m}} \mid 1 \leqslant j \leqslant n\right\}$, так что $1 \leqslant\left|U_{i d}\right| \leqslant n$. Поскольку $n$ ! необратимо в $R$, заключаем, что

$$
\sum_{u \in\left\{\sigma(f) \mid \sigma \in S_{n}\right\}} u
$$

есть полином над $R$ от $v_{s_{1}, \ldots, s_{m}}, 0 \leqslant \sigma_{1}+\ldots+\sigma_{m} \leqslant \operatorname{deg} f$. Это завершает доказательство.

Пусть $S_{n}$ - симметрическая группа степени $n \geqslant 2$ и

$$
v_{s_{1}, \ldots, s_{m}}=\sum_{j=1}^{n} x_{1 j}^{s_{1}} \ldots x_{m j}^{s_{m}}
$$

- однородный полином в $A_{m n}^{S_{n}}$ степени $s_{1}+\ldots+s_{m} \geqslant 1$. Пусть $R-$ поле простой характеристики $p$, делящей $n$. Следующий результат показывает, что оценка Вейля не выполняется при $m>n \geqslant 2$. 
Предложение 3. Пусть $R$ - поле простой харажтеристики $p$ u $S_{n}$ - симметрическая группа степени $n \geqslant 2$. Если р делит $n$ u $m>n$, то элемент $v_{11 \ldots 11} \in A_{m n}^{S_{n}}$ нельзя представить в виде полинома над $R$ от инвариантов

$$
u_{r_{1}, \ldots, r_{m}}, \quad 1 \leqslant r_{1}+\ldots+r_{m} \leqslant n .
$$

Доказателъство. Для доказательства от противного предположим, что $v_{11 . .11}$ можно представить в виде полинома над $R$ от $u_{r_{1}, \ldots, r_{m}}, 1 \leqslant r_{1}+\ldots+r_{m} \leqslant n$, и запишем $v_{11 \ldots 11}$ в виде

$$
v_{11 \ldots 11}=\sum_{s_{1}+2 s_{2}+\ldots+n s_{n}=m} a_{s_{1}, \ldots, s_{n}} \sum_{R_{m}\left(s_{1}, \ldots, s_{n}\right)} \prod_{\nu=1}^{n} \prod_{\sigma_{\nu}=1}^{s_{\nu}} u_{r_{1 \sigma_{\nu}}, \ldots, r_{m \sigma_{\nu}}}
$$

с некоторыми коэффициентами $a_{s_{1}, \ldots, s_{n}} \in R$, где $R_{m}\left(s_{1}, \ldots, s_{n}\right)$ - множество всех целых неотрицательных чисел $r_{i j}, i=1, \ldots, m, j=1, \ldots, s_{\nu}, \nu=1, \ldots, n$ таких, что

$$
\begin{aligned}
r_{1 \sigma_{\nu}}+\ldots+r_{m \sigma_{\nu}} & =\nu, \quad 1 \leqslant \sigma_{\nu} \leqslant s_{\nu}, \quad 1 \leqslant \nu \leqslant n \\
r_{i \sigma_{1}}+\ldots+r_{i \sigma_{n}} & =1, \quad 1 \leqslant i \leqslant m .
\end{aligned}
$$

Не теряя общности, можно предположить, что если $k \leqslant n-$ наименьшее положительное число такое, что $s_{k} \geqslant 1$, то

$$
r_{1 \sigma_{k}}= \begin{cases}1, & \text { если } \sigma_{k}=1 \\ 0, & \text { если } 2 \leqslant \sigma_{k} \leqslant s_{k}\end{cases}
$$

Дифференцируя приведенное выше равенство по $x_{11}$ и учитывая, что

$$
\frac{\partial u_{r_{1}, \ldots, r_{m}}}{\partial x_{11}}= \begin{cases}0 & \text { если } r_{1}=0 \\ u_{0, r_{2}, \ldots, r_{m}}^{(1,0, \ldots, 0)} & \text { если } r_{1}=1\end{cases}
$$

где $u_{0, r_{2}, \ldots, r_{m}}^{(1,0, \ldots, 0)}$ - соответствующий элементарный симметрический полином от векторов $\left(x_{2 j}, \ldots, x_{m j}\right), 1 \leqslant j \leqslant n$, получаем, что

$$
x_{21} \ldots x_{m 1}=\sum_{s_{1}+2 s_{2}+\ldots+n s_{n}=m} a_{s_{1}, \ldots, s_{n}} \sum_{j=1}^{n} \Psi_{s_{1}, \ldots, s_{n}}^{(j)}
$$

где

$$
\Psi_{s_{1}, \ldots, s_{n}}^{(j)}=\sum_{R_{m}\left(s_{1}, \ldots, s_{n}\right)} u_{0, r_{2 \sigma_{k}}, \ldots, r_{m \sigma_{k}}}^{(1,0, \ldots, 0)} \prod_{\sigma_{k}=2}^{s_{k}} u_{0, r_{2 \sigma_{k}}, \ldots, r_{m \sigma_{k}}} \prod_{\nu=1, \nu \neq k}^{n} \prod_{\sigma_{\nu}=1}^{s_{\nu}} u_{0, r_{2 \sigma_{\nu}} \ldots, r_{m \sigma_{\nu}}}
$$

Обозначим $\omega_{0, r_{2}, \ldots, r_{m}}$ значение $u_{0, r_{2}, \ldots, r_{m}}$ в точке

$$
\left(x_{11}, \ldots, x_{m 1} ; \ldots ; x_{1 n}, \ldots, x_{m n}\right)=(1, \ldots, 1 ; \ldots ; 1 \ldots, 1) .
$$

Поскольку $m>n \geqslant 2$, каждая двоичная последовательность $\left(0, r_{2}, \ldots, r_{m}\right)$, входящая в последнее равенство, содержит $l$ ненулевых членов для некоторого $1 \leqslant l \leqslant n$. В этом случае

$$
\omega_{0, r_{2}, \ldots, r_{m}}=n(n-1) \ldots(n-l+1),
$$

2 Дискретная математика, т.12 №4 
и полагая в (2)

$$
x_{11}=\ldots=x_{m 1}=\ldots=x_{1 n}=\ldots=x_{m n}=1,
$$

получаем соотношение

$$
1=n \sum_{s_{1}+2 s_{2}+\ldots+n s_{n}=m} b_{s_{1}, \ldots, s_{n}},
$$

невозможное в $R$ для любого простого числа $p$, делящего $n$. Это доказывает предложение 3.

\section{3. Доказательство теоремы 1}

Пусть $S_{n}$ - симметрическая группа степени $n$. Предположим, что $f$ - одночлен в $A_{m n}$ и $w \in A_{m n}^{S_{n}}$ - полиномиальный инвариант относительно $S_{n}$. Поскольку $\sigma(w)=w$ для любого $\sigma \in S_{n}$, полиномы $w$ и $\sigma(w)$ имеют одинаковые коэффициенты. Это показывает, что любой полиномиальный инвариант в $S_{n}$ является $R$-линейной комбинацией инвариантов

$$
v=\sum_{u \in\left\{\sigma(f) \mid \sigma \in S_{n}\right\}} u,
$$

где $f$ пробегает одночлены, появляющиеся в $w$.

Пусть $\left(i_{1}, \ldots, i_{\mu}\right)$ - последовательность элементов $i_{1}, \ldots, i_{\mu} \in\{1, \ldots, n\}$. Вначале докажем, что каждый инвариант $w_{\mu}$ вида

$$
w_{\mu}=\sum_{j=1}^{n} x_{i_{1}, j} \ldots x_{i_{\mu}, j}
$$

является полиномом над $R$ от поляризованных элементарных симметрических полиномов

$$
u_{r_{1}, \ldots, r_{m}}, \quad 1 \leqslant r_{1}+\ldots+r_{m} \leqslant n .
$$

Если $\mu \leqslant n+1$, то утверждение теоремы 1 следует из предложения 1 . Предположим теперь, что $\mu>n+1$, и проведем доказательство индукцией по $\mu$. Пусть

$$
\tilde{x}_{i_{s}, j}= \begin{cases}x_{i_{s}, j}, & \text { если } s \leqslant n, \\ x_{i_{n+1}, j} x_{i_{n+2}, j} \ldots x_{i_{\mu+1}, j}, & \text { если } s=n+1,\end{cases}
$$

при $j=1,2, \ldots, n$, тогда

$$
w_{\mu+1}=\sum_{j=1}^{n} \tilde{x}_{i_{1}, j} \ldots \tilde{x}_{i_{n}, j} \tilde{x}_{i_{n+1}, j}
$$

Пусть

$$
\tilde{A}_{m n}=R\left[\tilde{x}_{11}, \ldots, \tilde{x}_{m 1} ; \ldots ; \tilde{x}_{1 n}, \ldots, \tilde{x}_{m n}\right]
$$

и $\tilde{A}_{m n}^{S_{n}}-$ подалгебра инвариантов алгебры $\tilde{A}_{m n}$. Из следствия 5 вытекает, что $w_{\mu+1}$ - полином над $R$ от поляризованных элементарных симметрических полиномов

$$
\tilde{u}_{r_{1}, \ldots, r_{m}} \in \tilde{A}_{m n}, \quad 1 \leqslant r_{1}+\ldots+r_{m} \leqslant n .
$$


Так как каждый полином $\tilde{u}_{r_{1}, \ldots, r_{m}}$ имеет вид

$$
\tilde{u}_{r_{1}, \ldots, r_{m}}=\sum_{\tilde{u} \in\{\tau(\tilde{f}) \mid \tau \in G\}} \tilde{u},
$$

для некоторого одночлена $\tilde{f} \in \tilde{A}_{m n}$ степени, не большей $n$, согласно предложению 1 он может быть записан в виде полинома над $R$ от инвариантов

$$
\tilde{v}_{s_{1}, \ldots, s_{m}}=\sum_{j=1}^{n} \tilde{x}_{1 j}^{s_{1}} \ldots \tilde{x}_{m j}^{s_{m}}
$$

степени, не большей $n$. Кроме того, каждый инвариант $\tilde{v}_{s_{1}, \ldots, s_{m}}$ имеет вид

$$
\tilde{v}_{s_{1}, \ldots, s_{m}}=\sum_{j=1}^{n} x_{1 j}^{t_{1}} \ldots x_{m j}^{t_{j}}
$$

где $1 \leqslant t_{1}+\ldots+t_{m} \leqslant \mu$. По предположению индукции каждый инвариант $\tilde{v}_{s_{1}, \ldots, s_{m}}$ является полиномом над $R$ от

$$
u_{r_{1}, \ldots, r_{m}}, \quad 1 \leqslant r_{1}+\ldots+r_{m} \leqslant n
$$

поэтому $w_{\mu+1}$ - также полином над $R$ от поляризованных элементарных симметрических полиномов $u_{r_{1}, \ldots, r_{m}}, 1 \leqslant r_{1}+\ldots+r_{m} \leqslant n$.

Для завершения доказательства заметим, что в силу предложения 2 каждый элемент $v \in A_{m n}^{S_{n}}$ можно записать в виде полинома над $R$ от инвариантов $w_{\mu}$.

\section{4. Доказательство теоремы 3}

Рассуждения, используемые при доказательстве теоремы 3 , те же, что и в доказательстве предложения 3 . Пусть $S_{n}$ - симметрическая группа степени $n \geqslant 2$ и $p-$ простой делитель $n$. Пусть $R$ - поле характеристики $p$ и $A_{m n}^{S_{n}}$ - алгебра векторных инвариантов над $R$ относительно $S_{n}$. Пусть

$$
v_{s_{1}, \ldots, s_{m}}, \quad s_{1}+\ldots+s_{m} \geqslant 1
$$

обозначает полином в $A_{m n}^{S_{n}}$ вида

$$
v_{s_{1}, \ldots, s_{m}}=\sum_{j=1}^{n} x_{1 j}^{s_{1}} \ldots x_{m j}^{s_{m}} .
$$

Напомним, что каждый векторный инвариант $v \in A_{m n}^{S_{n}}$ является $R$-линейной комбинацией инвариантов

$$
w=\sum_{u \in\left\{\sigma(f) \mid \sigma \in S_{n}\right\}} u,
$$

где $f$ пробегает одночлены из $A_{m n}$, встречающиеся в $v$.

Для доказательства теоремы 3 , достаточно показать, что если $p^{\alpha}$ делит $n$, то каждая система образующих $R$-алгебры $A_{m n}^{S_{n}}$ содержит хотя бы один порождающий элемент $v$ степени $m\left(p^{\alpha}-1\right)$. Главным является то, что инвариант $v_{p^{\alpha}-1, \ldots, p^{\alpha}-1}$ 
степени $m\left(p^{\alpha}-1\right)$ не может быть представлен в виде полинома над $R$ от векторных инвариантов меньшей степени.

Обозначим $W_{m n}^{S_{n}}$ множество образующих $R$-алгебры $A_{m n}^{S_{n}}$ вида

$$
w=\sum_{u \in\left\{\sigma(f) \mid \sigma \in S_{n}\right\}} u,
$$

где $f$ - одночлен в $A_{m n}$ степени, меньшей $m\left(p^{\alpha}-1\right)$. Каждый векторный инвариант $v \in A_{m n}^{S_{n}}$, степень которого меньше, чем $m\left(p^{\alpha}-1\right)$, можно представить в виде $R$-линейной комбинации элементов $w \in W_{m n}^{S_{n}}$, поэтому достаточно доказать, что инвариант $v_{p^{\alpha}-1, \ldots, p^{\alpha}-1}$ непредставим в виде полинома над $R$ от элементов $w \in W_{m n}^{S_{n}}$. Пусть $l$ - мощность $W_{m n}^{S_{n}}$. Занумеруем элементы множества $W_{m n}^{S_{n}}$ числами $1,2, \ldots, l$ и предположим, чтобы прийти к противоречию, что инвариант $v_{p^{\alpha}-1, \ldots, p^{\alpha}-1}$ является полиномом над $R$ от $w_{1}, \ldots, w_{l}$, то есть

$$
v_{p^{\alpha}-1, \ldots, p^{\alpha}-1}=\sum_{M} a_{\mu_{1}, \ldots, \mu_{l}} w_{1}^{\mu_{1}} \ldots w_{l}^{\mu_{l}}, \quad a_{\mu_{1}, \ldots, \mu_{l}} \in R
$$

где $M-$ множество всех неотрицательных целых чисел $\mu_{1}, \ldots, \mu_{l}$ таких, что

$$
\begin{aligned}
0 & \leqslant \mu_{1}+\ldots+\mu_{l} \leqslant m\left(p^{\alpha}-1\right), \\
\mu_{1} \operatorname{deg} w_{1}+\ldots+\mu_{l} \operatorname{deg} w_{l} & =m\left(p^{\alpha}-1\right) .
\end{aligned}
$$

Сравнивая степени одночленов, появляющихся в обеих сторонах последнего равенства (относительно каждой из переменных $x_{11}, \ldots, x_{m 1} ; \ldots ; x_{1 n}, \ldots, x_{m n}$ ), находим, что $\mu_{k} \in\left\{0,1, \ldots, p^{\alpha}-1\right\}, 1 \leqslant k \leqslant l$, и $\mu_{1}+\ldots+\mu_{l}>1$; кроме того, каждый инвариант

$$
w_{k}=\sum_{u \in\left\{\sigma\left(f_{k}\right) \mid \sigma \in S_{n}\right\}} u,
$$

который появляется в правой части с ненулевым коэффициентом, порождается одночленом $f_{k} \in A_{m n}$ вида

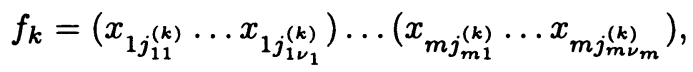

где $1 \leqslant j_{i j_{i 1}}^{(k)} \leqslant \ldots \leqslant j_{i \nu_{i}^{(k)}} \leqslant n$ и $j_{i \nu_{i}^{(k)}} \leqslant p^{\alpha}-1$ хотя бы для одного $i, i=1,2, \ldots, m$.

Обозначим $\omega_{k}$ значение $w_{k}$ в точке

$$
\left(x_{11}, \ldots, x_{m 1} ; \ldots ; x_{1 n}, \ldots, x_{m n}\right)=(1, \ldots, 1 ; \ldots ; 1, \ldots, 1)
$$

и заметим, что $\omega_{k}=\left|\operatorname{orb}\left(f_{k}\right)\right|$, где

$$
\operatorname{orb}\left(f_{k}\right)=\left\{\sigma\left(f_{k}\right) \mid \sigma \in S_{n}\right\}
$$

есть орбита $f_{k}$ относительно $S_{n}$. Если

$$
S_{n}\left(f_{k}\right)=\left\{\sigma \in S_{n} \mid \sigma\left(f_{k}\right)=f_{k}\right\}
$$

является группой изотропии $f_{k}$, то

$$
\left|\operatorname{orb}\left(f_{k}\right)\right|=\frac{\left|S_{n}\right|}{\left|S_{n}\left(f_{k}\right)\right|}=\frac{n !}{\left|S_{n}\left(f_{k}\right)\right|} .
$$


Поскольку $j_{i \nu_{i}^{(k)}} \leqslant p^{\alpha}-1$ для каждого $k=1,2, \ldots, l$ и хотя бы для одного $i$, $i=1,2, \ldots, m$, показатель степени $p$ в разложении на простые множители каждого из чисел $\left|S_{n}\left(f_{k}\right)\right|$ меньше, чем показатель степени $p$ в разложении $n$ !. Отсюда следует, что $\left|\operatorname{orb}\left(f_{k}\right)\right|$ делится на $p$, и поэтому $\omega_{k}=0$ в $R$ для всех $k=1,2, \ldots, l$. Дифференцируя тождество (3) по $x_{11}$, полагая

$$
x_{11}=\ldots=x_{m 1}=\ldots=x_{1 n}=\ldots=x_{m n}=1
$$

в получающемся равенстве и учитывая, что каждое произведение

$$
w_{1}^{\mu_{1}} \ldots w_{l}^{\mu_{l}}
$$

в правой части (3) содержит по крайней мере два множителя, скажем $w_{k}$ и $w_{k^{\prime}}$, $1 \leqslant k \leqslant k^{\prime} \leqslant l$, приходим к равенству $p-1=0$, что невозможно в $R$.

Инвариант $v_{p^{\alpha}-1, \ldots, p^{\alpha}-1}$ нельзя представить в виде полинома над $R$ от векторных инвариантов меньшей степени, поэтому каждая система образующих $R$-алгебры $A_{m n}^{S_{n}}$ должна содержать хотя бы один порождающий элемент степени $m\left(p^{\alpha}-1\right)$. Замечая теперь, что каждая система образующих $R$-алгебры $A_{m n}^{S_{n}}$ содержит порождающий элемент степени $n$ (например, инвариант $w=x_{11} \ldots x_{1 n}$ ), находим, что она содержит порождающий элемент $v$ степени не меньшей $\max \left\{n, m\left(p^{\alpha}-1\right)\right\}$.

\section{5. Доказательство следствия 3}

Пусть $R$ - коммутативное кольцо с единичным элементом $1, p-$ простой делитель $n$ ! и $I-$ максимальный идеал в $R$, содержащий $p$. Тогда $F=R / I-$ поле характеристики $p$. Рассмотрим гомоморфизм

$$
\varphi: R\left[x_{11}, \ldots, x_{m 1} ; \ldots ; x_{n 1}, \ldots, x_{m n}\right] \rightarrow F\left[x_{11}, \ldots, x_{m 1} ; \ldots ; x_{n 1}, \ldots, x_{m n}\right]
$$

который оставляет на месте все переменные $x_{i j}, 1 \leqslant i \leqslant m, 1 \leqslant j \leqslant n$. Этот гомоморфизм индуцирует сюръективный гомоморфизм

$$
\psi: R\left[x_{11}, \ldots, x_{m 1} ; \ldots ; x_{n 1}, \ldots, x_{m n}\right]^{S_{n}} \rightarrow F\left[x_{11}, \ldots, x_{m 1} ; \ldots ; x_{1 n}, \ldots, x_{m n}\right]^{S_{n}}
$$

Таким образом, $\psi$ отображает каждое множество образующих

$$
R\left[x_{11}, \ldots, x_{m 1} ; \ldots ; x_{1 n}, \ldots, x_{m n}\right]^{S_{n}}
$$

в множество образующих

$$
F\left[x_{11}, \ldots, x_{m 1} ; \ldots ; x_{1 n}, \ldots, x_{m n}\right]^{S_{n}}
$$

Из этого факта и следствия 2 заключаем, что каждая система образующих $R$ алгебры $R\left[x_{11}, \ldots, x_{m 1} ; \ldots ; x_{1 n}, \ldots, x_{m n}\right]^{S_{n}}$ содержит порождающий элемент степени, не меньшей $\max \{n, m(p-1)\}$. В частности, если $n=p$ - простое число, то она содержит порождающий элемент, степень которого не меньше, чем $\max \{n, m(n-1)\}$. 


\section{Список литературы}

1. Benson D. J., Polynomial invariants of finite groups. Cambridge Univ. Press, Cambridge, 1993.

2. Bourbaki N., Elements of mathematics, Algebra II. Springer, Berlin, 1990.

3. Campbell H. E. A., Hughes I., Pollack R. D., Vector invariants of symmetric groups, Canad. Math. Bull. (1990) 33, 391-397.

4. Dieudonne J. A., Carrel J. B., Invariant theory, old and new. Academic Press, New York, 1971.

5. Fleischmann P., A new degree bound for vector invariants of symmetric groups. Trans. Amer. Math. Soc. (1998) 350, 1703-1712.

6. Hilbert D., Über die vollen Invariantensystem. Math. Ann. (1893) 42, 313-373.

7. Kemper G., Lower degree bounds for modular invariants and a question of I. Hughes. Transformation Groups (1998) 3, 135-144.

8. Noether E., Der Endlichkeitssatz der Invarianten endlicher Gruppen. Math. Ann. (1916) 77, 89-92.

9. Noether E., Der Endlichkeitssatz der Invarianten endlicher linearer Gruppen der Charakteristik p. Nachr. Ges. Wiss. Göttingen (1926), 28-35.

10. Richman D. R., Invariants of finite groups over fields of characteristic p. Adv. Math. (1996) 124, 25-48.

11. Richman D. R., Explicit generators of the invariants of finite groups. Adv. Math. (1996) 124, 49-76.

12. Smith L., Polynomial invariants of finite groups. A. K. Peters, Wellesley, MA, 1995.

13. Smith L., Polynomial invariants of finite groups. A survey of recent developments. Bull. Amer. Math. Soc. (1997) 34, 211-250.

14. Stepanov S. A., Transcendence bases of the algebra of vector invariants for a symmetric group. Proc. Intern. Conf. Number Theory, Berlin, 1999, 487-501.

15. Степанов С. А., Полиномиальные инварианты конечных групп над полями простой характеристики. Дискретная математика (1999) 11, 3-14.

16. Waring E., Meditationes Algebraicae. Cambridge Univ. Press, Cambridge, 1782.

17. Weyl H., The classical groups, their invariants and representations. New Jersey, 1939.

Статья поступила 18.08.2000. 